\title{
A Simple and Efficient Power Flow for Distribution Networks
}

\author{
Yogambari Venkatesan $^{1}$ (1) $\cdot$ Arun Nambi Pandian ${ }^{1} \cdot$ Aravindhababu Palanivelu $^{1}$
}

Received: 19 December 2020 / Accepted: 19 January 2022 / Published online: 23 February 2022

(c) The Author(s), under exclusive licence to Springer Nature Singapore Pte Ltd. 2022

\begin{abstract}
This paper presents an efficient power flow (PF) for distribution networks (DN). The proposed PF method uses basic circuit laws in deriving the final PF expression and appears like the classical Gauss-Seidel PF algorithm of transmission systems. It possesses the advantages of forward and backward sweeps (FBS) based PF methods but avoids the FBS and formation of a Jacobian matrix. It primarily depends on a constant transformation matrix, formed only once from the network topology and feeder parameters. The transformation matrix relates the node currents with effective feeder voltage drops, and helps to compute the node voltages directly from the given set of load powers. The proposed PF was studied on 15, 33 and 69 node DNs, and the study exhibited that the performances in terms of accuracy, robustness to different $\mathrm{r} / \mathrm{x}$ ratios of distribution lines and computational efficiency of the proposed method are superior to those of existing methods.
\end{abstract}

Keywords Distribution power flow $\cdot$ Forward and backward sweeps $\cdot$ Compensation based power flow $\cdot$ Distribution management systems

\section{Introduction}

The concept of smart-grid for distribution networks (DN) has attracted many developing countries and steps have been initiated to modernize the existing DNs with advanced metering, distribution automation and distributed energy sources (DES). Such smart DNs require repeated and fast power flow (PF) solutions for performing various distribution management functions such as VAR control, load curtailment, DES placement, reconfiguration and so on. The PF techniques such as Gauss-Seidel, Newton-Raphson (NR), fast decoupled $\mathrm{PF}$, etc. are not suitable for DNs, as DNs are ill-conditioned and different from transmission systems in respect of network topology, feeder parameters and operating voltages [24].

$\mathrm{PF}$ is a computational procedure of determining steady state node voltages at fundamental frequency for a given load powers of DNs. The essential requirements of a PF method are fast convergence, lower memory requirement, lower computation time, and robust to wide variations in feeder parameters. Considering these requirements, several PF techniques have been suggested in recent years. These techniques are

Yogambari Venkatesan

yogambari26@gmail.com

1 Department of Electrical Engineering, Annamalai University, Annamalainagar, Tamil Nadu, India divided into node based and branch based techniques. The node based techniques usually solve a set of linearized PF equations involving Jocabian matrices for voltage corrections iteratively, while branch based techniques solve a set of basic circuit laws for steady state voltages by forward and backward sweeps (FBS) without involving Jacobian matrices. A compensation based PF (CPF) adapting FBS was suggested for DNs $[21,28]$. A G-matrix based decoupled PF (GDPF) using equivalent load currents was presented for DNs [18, 26]. A feeder-to-bus matrix based PF for DNs for eliminating FBSs by modelling the PF equations in compact form was outlined $[6,30]$. The PF of DNs was formulated as a convex optimisation problem and interior point algorithm was applied for solving the tailored problem for overcoming numerical ill-conditioning issues [14].

A loop-based PF was presented for 3-phase DNs [32]. A rotation based decoupled PF (RDPF) that eliminates the off-diagonal sub-Jacobian matrices by multiplying the PF equations with feeder's admittance angles was proposed for DNs [4]. A robust PF using the topology of unbalanced DNs was explained for eliminating matrix inversion and FBSs [2]. A PF adapting fuzzy logic for DNs with composite loads was developed for modelling the uncertain data [15]. A graph theory based 3-phase PF adapting a loop as reference unlike node reference was described [11]. A robust line current based decoupled PF (LDPF) was presented by discarding 
Fig. 1 Sample Network

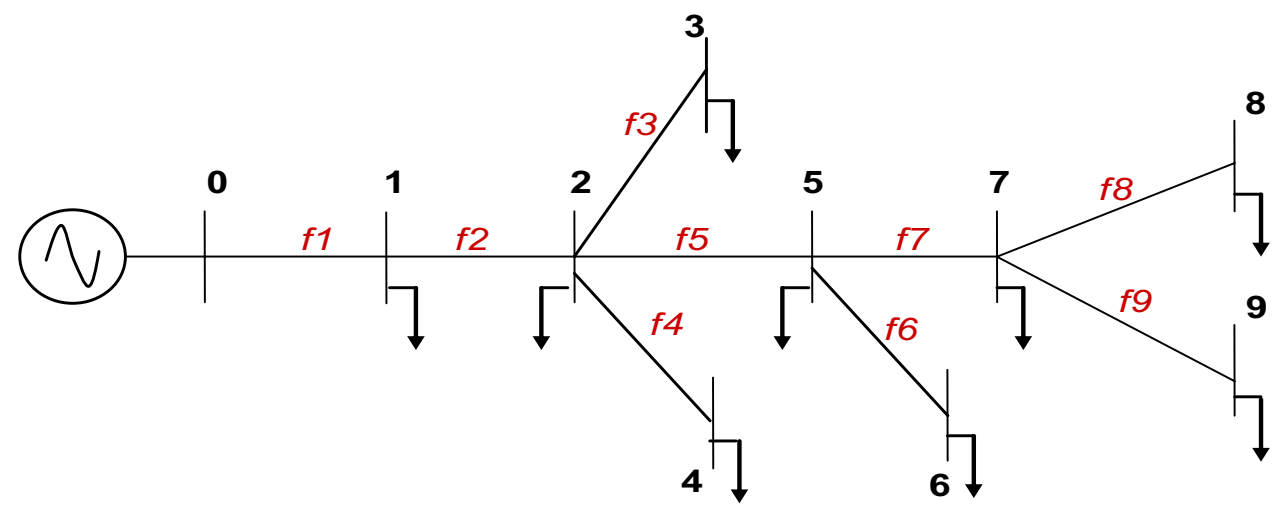

the off-diagonal sub-Jacobian matrices through transformations [5].

A net node power based PF for DNs was proposed in addition to presenting a sensible strategy for choosing starting voltages for achieving better convergence [25]. A distribution PF using basic laws was developed for studying many load models and different line r/x ratios [13]. Several existing PF methods of DNs were reviewed [17]. The NR and FBS schemes with changing load patterns and different line $\mathrm{r} / \mathrm{x}$ ratios were studied and portrayed that FBS scheme was better than NR technique [20]. An AC PF relating the voltages, feeder currents and powers was proposed for DNs. This method can be used as fundamental tool for developing several online distribution management functions [9].

An interactive software for carrying out the PF and DES placement in DNs was developed [1]. A Z-bus based 3-phase PF by modelling different models of loads and network parameters without considering the radial nature of DNs was suggested for DNs [8]. A PF method employing power loss derivatives with advantages of being non-iterative and efficient was outlined for DNs with DES [3]. A simple PF scheme adapting fixed matrices for relating load currents with line currents and then with bus voltages was proposed [31]. The convergence of bus-impedance matrix based PF methods of DNs was studied and an acceleration technique was suggested for enhancing the convergence of the PF [33]. The transmission and 3-phase DNs were mathematically modelled and applied for solving PF problems with advantage of realizing reliable convergence [23].

A compact modelling of DNs in phasor domain was formulated by altering the nodal equations, and used for iteratively solving the PF [19]. A simple PF technique was outlined for DNs comprising FACTS devices and DES plants [29]. A NR based PF technique suitable for ring DNs was developed [27]. Sequence components based three-phase PF was suggested for DNs with intent of lowering the dimension of the PF problem and simplifying the solution procedure
[12]. A PF method adapting simple circuit laws and equations was outlined for DNs [22].

Most of the FBS based methods calculate the load currents from the initial node voltages, and then successively compute the feeder currents starting from last feeder to first feeder, evaluate the feeder voltage drops, and update the node voltages from first node to last node. These calculations represent an iteration, and are repeated till convergence. While the Newton based methods require inversion/factorization of time consuming Jacobian matrix during iterations, and they may result oscillatory convergence or divergence due to radial nature, wide varying feeder $\mathrm{r} / \mathrm{x}$ ratios and lower operating voltages of the DN. The goal of this paper is to build a simple PF method for updating feeder voltage drops and node voltages directly from load currents, thereby avoiding recursive calculations of feeder currents, feeder voltage drops and node voltages, feeder by feeder or node by node. Besides, the paper endeavours to achieve the features of low computational burden, robust, and fast convergence. The prime contributions of the paper are:

- A simple and efficient PF method, derived from the basic circuit laws, has been developed for DNs.

- The method is based a constant transformation matrix [A] that directly transforms the nodes' load powers into net voltage drops between the source node and other nodes in the DN.

- The final PF equation is very simple and appears like Gauss-Seidel PF equation. It is solved iteratively for node voltages from the given set of load powers.

- The method does not require a Jacobian matrix and its time-consuming inversion.

- The method has been tested on three IEEE DNs for studying its accuracy, robustness and computational efficiency.

The paper is divided into four sections. Section 2 tailors the proposed PF; Section 3 discusses the results; and Section 4 outlines the findings. 


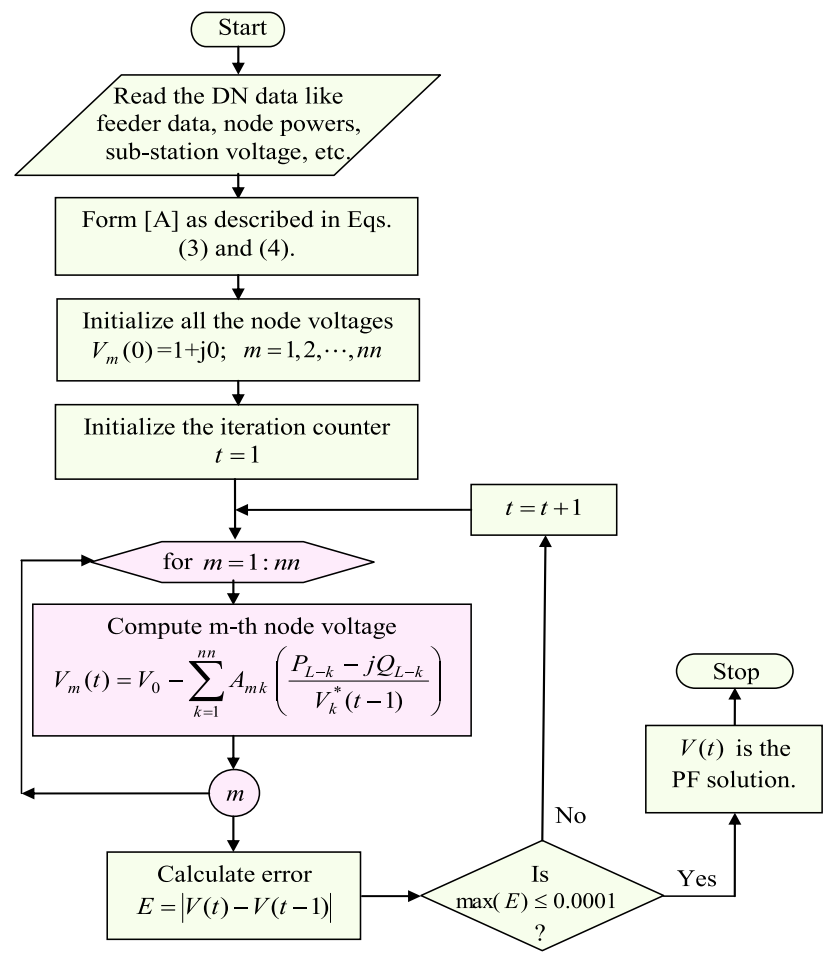

Fig. 2 Flowchart of the proposed PF

\section{Proposed Distribution Power Flow}

Figure 1 shows a sample 10-node DN, wherein the 0-th node represents the substation node and nine feeders serving the remaining load nodes. The net effective voltage drop $\left(v_{0 m}\right)$ across the feeders between node- 0 and other nodes $(m=1,2, \cdots, 9)$ can be computed from voltage drops at each feeder $\left(e_{m}\right)$ by Eq. (1).

$\left[\begin{array}{l}v_{01} \\ v_{02} \\ v_{03} \\ v_{04} \\ v_{05} \\ v_{06} \\ v_{07} \\ v_{08} \\ v_{09}\end{array}\right]=\left[\begin{array}{lllllllll}1 & 0 & 0 & 0 & 0 & 0 & 0 & 0 & 0 \\ 1 & 1 & 0 & 0 & 0 & 0 & 0 & 0 & 0 \\ 1 & 1 & 1 & 0 & 0 & 0 & 0 & 0 & 0 \\ 1 & 1 & 0 & 1 & 0 & 0 & 0 & 0 & 0 \\ 1 & 1 & 0 & 0 & 1 & 0 & 0 & 0 & 0 \\ 1 & 1 & 0 & 0 & 1 & 1 & 0 & 0 & 0 \\ 1 & 1 & 0 & 0 & 1 & 0 & 1 & 0 & 0 \\ 1 & 1 & 0 & 0 & 1 & 0 & 1 & 1 & 0 \\ 1 & 1 & 0 & 0 & 1 & 0 & 1 & 0 & 1\end{array}\right]\left[\begin{array}{l}e_{1} \\ e_{2} \\ e_{3} \\ e_{4} \\ e_{5} \\ e_{6} \\ e_{7} \\ e_{8} \\ e_{9}\end{array}\right]$

The voltage drop across each feeder $\left(e_{m}\right)$ can be calculated by multiplying the feeder impedance $\left(z_{m}\right)$ with feeder current $\left(I_{m}\right)$. Replacing the feeder voltage drop $\left(e_{m}\right)$ by the product of primitive impedance matrix comprising self impedance values $\left(z_{m}\right)$ at its diagonal locations and feeder currents $\left(I_{m}\right)$, Eq. (1) can be written as,

$\left[\begin{array}{l}v_{01} \\ v_{02} \\ v_{03} \\ v_{04} \\ v_{05} \\ v_{06} \\ v_{07} \\ v_{08} \\ v_{09}\end{array}\right]=\left[\begin{array}{lllllllll}1 & 0 & 0 & 0 & 0 & 0 & 0 & 0 & 0 \\ 1 & 1 & 0 & 0 & 0 & 0 & 0 & 0 & 0 \\ 1 & 1 & 1 & 0 & 0 & 0 & 0 & 0 & 0 \\ 1 & 1 & 0 & 1 & 0 & 0 & 0 & 0 & 0 \\ 1 & 1 & 0 & 0 & 1 & 0 & 0 & 0 & 0 \\ 1 & 1 & 0 & 0 & 1 & 1 & 0 & 0 & 0 \\ 1 & 1 & 0 & 0 & 1 & 0 & 1 & 0 & 0 \\ 1 & 1 & 0 & 0 & 1 & 0 & 1 & 1 & 0 \\ 1 & 1 & 0 & 0 & 1 & 0 & 1 & 0 & 1\end{array}\right]\left[\begin{array}{ccccccccc}z_{1} & 0 & 0 & 0 & 0 & 0 & 0 & 0 & 0 \\ 0 & z_{2} & 0 & 0 & 0 & 0 & 0 & 0 & 0 \\ 0 & 0 & z_{3} & 0 & 0 & 0 & 0 & 0 & 0 \\ 0 & 0 & 0 & z_{4} & 0 & 0 & 0 & 0 & 0 \\ 0 & 0 & 0 & 0 & z_{5} & 0 & 0 & 0 & 0 \\ 0 & 0 & 0 & 0 & 0 & z_{6} & 0 & 0 & 0 \\ 0 & 0 & 0 & 0 & 0 & 0 & z_{7} & 0 & 0 \\ 0 & 0 & 0 & 0 & 0 & 0 & 0 & z_{8} & 0 \\ 0 & 0 & 0 & 0 & 0 & 0 & 0 & 0 & z_{9}\end{array}\right]\left[\begin{array}{c}I_{1} \\ I_{2} \\ I_{3} \\ I_{4} \\ I_{5} \\ I_{6} \\ I_{7} \\ I_{8} \\ I_{9}\end{array}\right]$

The feeder currents $\left(I_{m}\right)$ can be calculated by summing the appropriate load currents $\left\{\left(P_{L-k}-j Q_{L-k}\right) / V_{k}^{*}\right\}$ through
Table 1 PF Solution obtained for 15 node DN

\begin{tabular}{|c|c|c|c|c|c|c|c|c|}
\hline \multirow[t]{2}{*}{ Node No } & \multicolumn{2}{|l|}{ PSDPF } & \multicolumn{2}{|l|}{ LDPF } & \multicolumn{2}{|l|}{ RDPF } & \multicolumn{2}{|l|}{$\mathrm{CPF}$} \\
\hline & $V$ & $\delta$ & $V$ & $\delta$ & $V$ & $\delta$ & $V$ & $\delta$ \\
\hline 0 & 1.0000 & 0.0000 & 1.0000 & 0.0000 & 1.0000 & 0.0000 & 1.0000 & 0.0000 \\
\hline 1 & 0.9713 & 0.0006 & 0.9713 & 0.0006 & 0.9713 & 0.0006 & 0.9713 & 0.0006 \\
\hline 2 & 0.9567 & 0.0009 & 0.9567 & 0.0009 & 0.9567 & 0.0009 & 0.9567 & 0.0009 \\
\hline 3 & 0.9509 & 0.0010 & 0.9509 & 0.0010 & 0.9509 & 0.0010 & 0.9509 & 0.0010 \\
\hline 4 & 0.9499 & 0.0012 & 0.9499 & 0.0012 & 0.9499 & 0.0012 & 0.9499 & 0.0012 \\
\hline 5 & 0.9484 & 0.0015 & 0.9484 & 0.0015 & 0.9484 & 0.0015 & 0.9484 & 0.0015 \\
\hline 6 & 0.9486 & 0.0015 & 0.9486 & 0.0015 & 0.9486 & 0.0015 & 0.9486 & 0.0015 \\
\hline 7 & 0.9500 & 0.0023 & 0.9500 & 0.0023 & 0.9500 & 0.0023 & 0.9500 & 0.0023 \\
\hline 8 & 0.9458 & 0.0032 & 0.9458 & 0.0032 & 0.9458 & 0.0032 & 0.9458 & 0.0032 \\
\hline 9 & 0.9445 & 0.0035 & 0.9445 & 0.0035 & 0.9445 & 0.0035 & 0.9445 & 0.0035 \\
\hline 10 & 0.9680 & 0.0013 & 0.9680 & 0.0013 & 0.9680 & 0.0013 & 0.9680 & 0.0013 \\
\hline 11 & 0.9669 & 0.0015 & 0.9669 & 0.0015 & 0.9669 & 0.0015 & 0.9669 & 0.0015 \\
\hline 12 & 0.9582 & 0.0033 & 0.9582 & 0.0033 & 0.9582 & 0.0033 & 0.9582 & 0.0033 \\
\hline 13 & 0.9560 & 0.0038 & 0.9560 & 0.0038 & 0.9560 & 0.0038 & 0.9560 & 0.0038 \\
\hline 14 & 0.9570 & 0.0036 & 0.9570 & 0.0036 & 0.9570 & 0.0036 & 0.9570 & 0.0036 \\
\hline
\end{tabular}


tracking the load current flow paths from source node. The above equation can be modified as, can be computed from the network topology and feeder impedances of the DN in advance prior to iterative PF com-

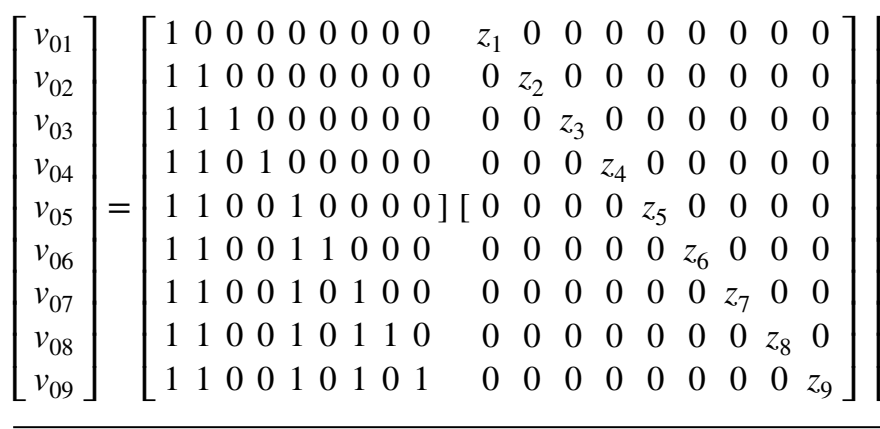

Where,

$P_{L-k}$ and $Q_{L-k}$ denotes the real and reactive load power at node-k respectively

$V_{m} \quad$ indicates the complex voltage at node-m.

It can be observed from Eq. (3) that the middle three matrices represent the topology of the DN and the feeder's self-impedance parameters. Besides the first and third matrices are just transpose of one another. Representing the product of three middle matrices as a constant transformation matrix [A] that transforms the node currents into effective feeder voltage drops, Eq. (3) can be written as,

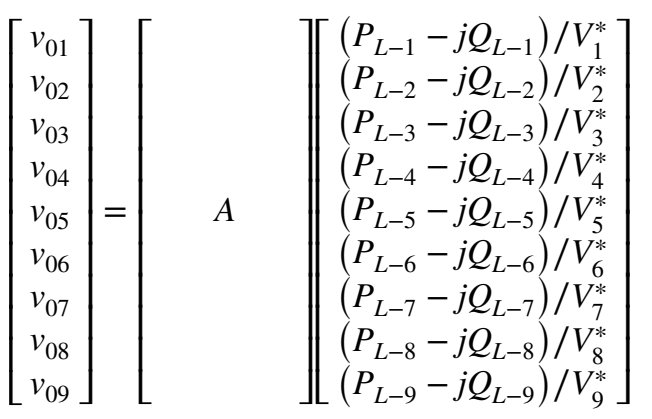

Eq. (4) can be written in compact form for a generalized DN as,

$v_{0 m}=\sum_{k=1}^{n n} A_{m k}\left(\frac{P_{L-k}-j Q_{L-k}}{V_{k}^{*}}\right) m=1,2, \cdots, n n$

where $n n$ denotes the number of nodes in the DN excluding sub-station node.

The node voltages at $t$-th iteration can be obtained by subtracting the effective feeder voltage drops from the source node voltage $\left(V_{0}\right)$ by Eq. (6).

$V_{m}(t)=V_{0}-\sum_{k=1}^{n n} A_{m k}\left(\frac{P_{L-k}-j Q_{L-k}}{V_{k}^{*}(t-1)}\right) m=1,2, \cdots, n n$

The solution process involves very simple procedure involving the formation of the constant matrix $[\mathrm{A}]$, which putations. Eq. (6) is solved for all node voltages repeatedly till the error between the voltages of two subsequent iterations are less than a small tolerance value (0.0001). The equation appears like the Gauss-Seidel PF expression of transmission systems but offers good convergence unlike Gauss-Seidel algorithm. The algorithmic steps of the developed method are presented in Fig. 2.

\section{Simulation Results}

The proposed simple distribution PF (PSDPF) has been tested on IEEE 15, 33 and 69 node DNs [7, 10, 16] using Matlab-2016 software in a $2.67 \mathrm{GHz}$ Intel core-i5 computer. The PF solution and other performances of PSDPF have been compared with three existing PF methods of LDPF [5], RDPF [4] and CPF [28]. The maximum difference in voltages of two subsequent iterations has been compared with a tolerance of 0.0001 per unit for performing convergence check.

Accuracy The final solution of the PSDPF has been found to be same as those of LDPF, RDPF and CPF for all the three test systems under study. To illustrate this, the PF solution of the PSDPF is compared only for 15-node DN with other methods in Table 1. It is very clear from the table that the PSDPF provides identical solution as those of existing methods, thereby exhibiting that the method is as accurate as any other PF method. The voltage solutions offered by the PSDPF for all the three test DNs are graphically plotted in Fig. 3.

Robustness The robustness of the PSDPF against different $\mathrm{r} / \mathrm{x}$ ratios of distribution feeders has been studied by varying $r$ while retaining $x$ constant and vice-versa. The number of iterations required for convergence of the PSDPF for different resistance and reactance values are compared with those of LDPF, RDPF and CPF methods. It is observed from the table that the number of iterations of PSDPF is same as that 
Fig. 3 Voltage solution of PSDPF

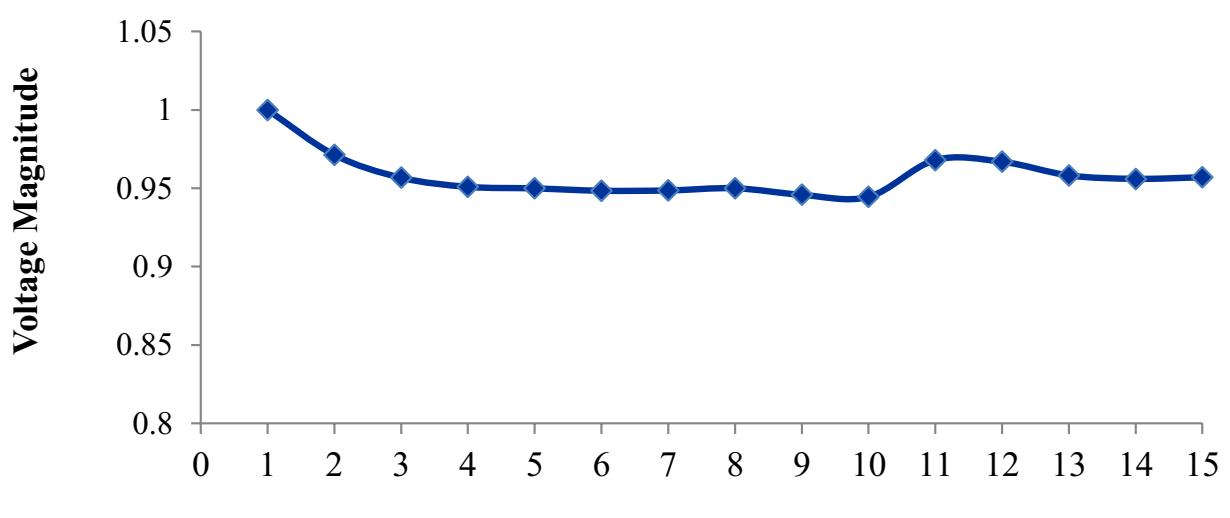

(a) 15 node DN

Node Numbers

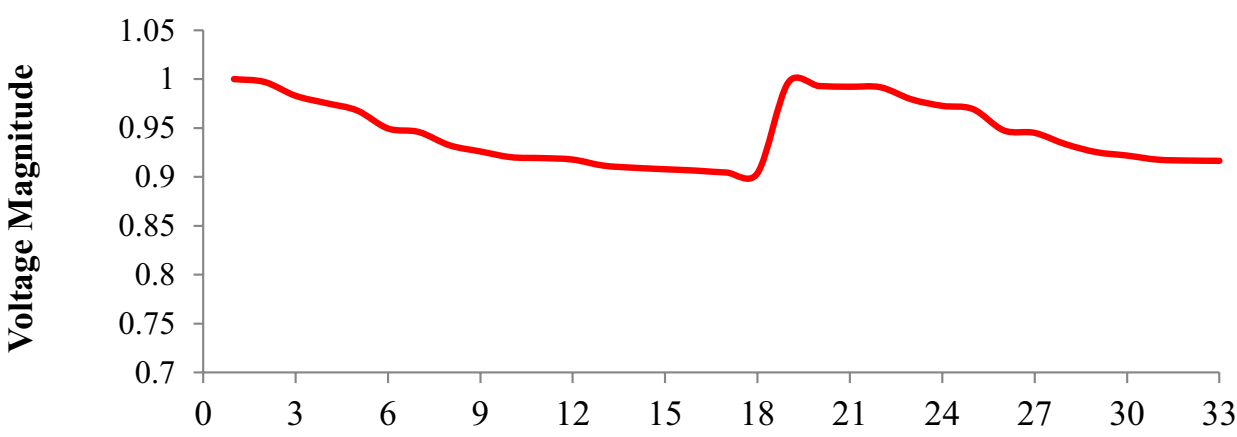

(b)33 node DN

Node Numbers

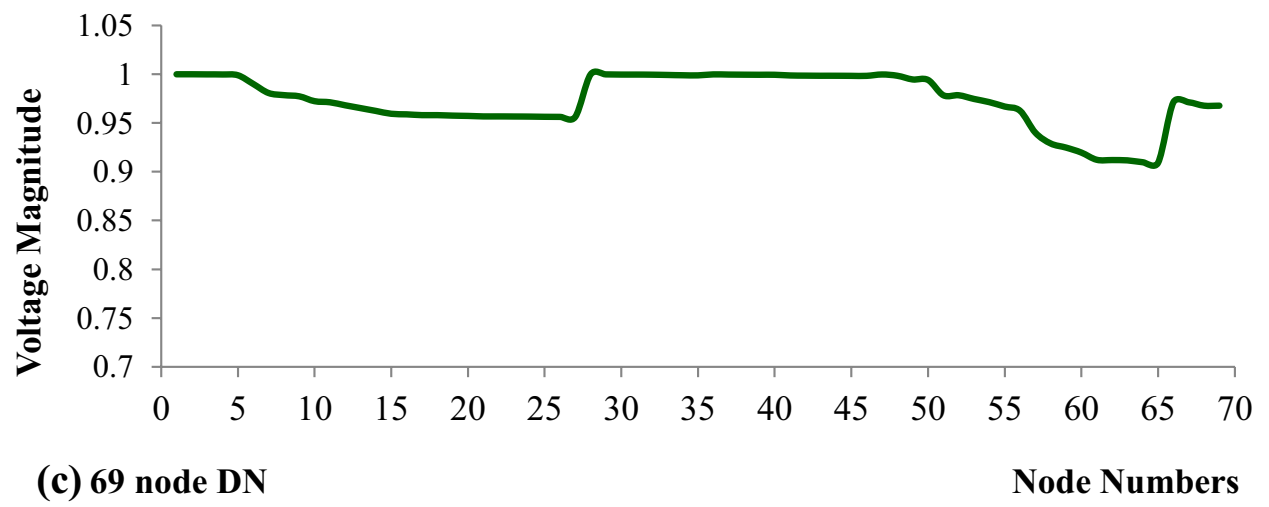

of CPF, and differs hardly by one iteration with those of LDPF and RDPF Table 2.

Computational Efficiency To study the computational efficiency of the PSDPF, all the methods under study were executed on the same $2.66 \mathrm{GHz}$ Intel core-i3 personal computer and their execution times were measured using the Matlab commands "tic" and "toc". Table 3 presents average execution time (AET) taken by all the methods and shows that the PSDPF takes very less AET than those of other methods. The table also presents the relative computational speed taking the inefficient RDPF method as reference. Analyzing the computation speed, it is very clear that PSDPF is $3.46,1.54$ and 1.84 times faster than that of RDPF for 15, 33 and 69 node DN respectively, thereby exhibiting its superior computational efficiency. If the method is executed on a modern 2.933/3.2 MHz Intel i5/i7 personal computers, the speed of computations will be at least 2-4 times faster than the speed presented in Table 3. With such high computational speed, the operational engineer at distribution management systems can interact online with the computer for studying the effects of sudden disconnection of a feeder, improvements due to inclusion of a shunt capacitor at a node, effects of load shedding, enhancements due to alteration in feeder configuration, 
Table 2 Number of Iterations

\begin{tabular}{|c|c|c|c|c|c|c|c|c|c|c|c|c|}
\hline \multirow[t]{2}{*}{$r+j x$} & \multicolumn{4}{|l|}{15 node } & \multicolumn{4}{|l|}{33 node } & \multicolumn{4}{|l|}{69 node } \\
\hline & PSDPF & LDPF & RDPF & CPF & PSDPF & LDPF & RDPF & $\mathrm{CPF}$ & PSDPF & LDPF & RDPF & $\mathrm{CPF}$ \\
\hline $0.05 r+\mathrm{j} 1.0 x$ & 3 & 4 & 3 & 3 & 5 & 5 & 5 & 5 & 3 & 4 & 4 & 3 \\
\hline $0.1 r+\mathrm{j} 1.0 x$ & 3 & 4 & 3 & 3 & 5 & 5 & 5 & 5 & 3 & 4 & 4 & 3 \\
\hline $0.2 r+\mathrm{j} 1.0 x$ & 3 & 4 & 3 & 3 & 5 & 6 & 5 & 5 & 4 & 4 & 4 & 4 \\
\hline $0.6 r+\mathrm{j} 1.0 x$ & 3 & 4 & 3 & 3 & 5 & 6 & 5 & 5 & 4 & 5 & 4 & 4 \\
\hline $1.0 r+\mathrm{j} 1.0 x$ & 4 & 4 & 4 & 4 & 5 & 7 & 5 & 5 & 5 & 6 & 5 & 5 \\
\hline $1.4 r+\mathrm{j} 1.0 x$ & 4 & 5 & 4 & 4 & 7 & 9 & 7 & 7 & 6 & 7 & 6 & 6 \\
\hline $1.8 r+\mathrm{j} 1.0 x$ & 4 & 5 & 4 & 4 & 9 & 9 & 8 & 9 & 7 & 9 & 7 & 7 \\
\hline $2.0 r+\mathrm{j} 1.0 x$ & 4 & 5 & 4 & 4 & 9 & 9 & 9 & 9 & 7 & 9 & 7 & 8 \\
\hline $1.0 r+\mathrm{j} 2.0 x$ & 4 & 5 & 4 & 4 & 9 & 9 & 8 & 9 & 5 & 7 & 6 & 5 \\
\hline $1.0 r+\mathrm{j} 1.8 x$ & 4 & 5 & 4 & 4 & 8 & 9 & 8 & 8 & 5 & 7 & 6 & 5 \\
\hline $1.0 r+\mathrm{j} 1.4 x$ & 4 & 5 & 4 & 4 & 6 & 8 & 6 & 6 & 5 & 6 & 5 & 5 \\
\hline $1.0 r+\mathrm{j} 0.6 x$ & 3 & 4 & 3 & 3 & 6 & 6 & 5 & 6 & 5 & 6 & 5 & 5 \\
\hline $1.0 r+\mathrm{j} 0.2 x$ & 3 & 4 & 3 & 3 & 5 & 6 & 5 & 5 & 5 & 6 & 5 & 5 \\
\hline $1.0 r+\mathrm{j} 0.1 x$ & 3 & 4 & 3 & 3 & 5 & 6 & 5 & 5 & 5 & 6 & 5 & 5 \\
\hline $1.0 r+\mathrm{j} 0.05 x$ & 3 & 4 & 3 & 3 & 5 & 6 & 5 & 5 & 5 & 6 & 5 & 5 \\
\hline
\end{tabular}

Table 3 Computational Efficiency

\begin{tabular}{|c|c|c|c|c|c|c|c|c|}
\hline \multirow[t]{2}{*}{$\mathrm{DN}$} & \multicolumn{4}{|c|}{ Average Execution Time (milliseconds) } & \multicolumn{4}{|c|}{ Relative Computational Speed } \\
\hline & PSDPF & LDPF & RDPF & $\mathrm{CPF}$ & PSDPF & LDPF & RDPF & $\mathrm{CPF}$ \\
\hline 15 node & 4.33 & 6.82 & 15 & 5.71 & 3.46 & 2.20 & 1 & 2.63 \\
\hline 33 node & 10.64 & 14.53 & 16.4 & 11.82 & 1.54 & 1.13 & 1 & 1.39 \\
\hline 69 node & 18.58 & 31 & 34.27 & 22.99 & 1.84 & 1.11 & 1 & 1.49 \\
\hline
\end{tabular}

and so on, and take quick decisions for effective operation of the DNs.

The results explained in this section portray the superiority of PSDPF in respect of accuracy, computational efficiency and robustness to changes in feeder parameters, and illustrate its suitability in online environment of distribution management systems.

\section{Conclusions}

A simple and efficient PF method, derived from the basic circuit laws, for DNs was proposed. The method did not require formation of a Jacobian matrix but was based on a constant matrix [A], which directly relates the node voltages with load powers and makes the solution procedure simple. The method inherited the features of FBS based methods and took lower computational time. The final equation looked like the GaussSeidel PF expression of transmission systems but did not possess the drawbacks of Gauss-Seidel approach. The method was tested on three IEEE DNs, and the results exhibited that the method offered good accuracy, robustness to changes in feeder $\mathrm{r} / \mathrm{x}$ ratios, and good computational efficiency. The proposed method can thus be well employed for practical online applications in distribution management systems. The limitation is that the method cannot be applied on studying transmission networks. The method can further be modified to perform PF of DNs with DES and FACTS devices. Besides, the method can be integrated with reconfiguration and optimal FACTS placement problems.

Data Availability Statement Data sharing is not applicable to this article as no new data were created or analyzed in this study.

\section{References}

1. Akorede MF, Hizam H, Aris I, Ab Kadir MZ, Hojabri M (2017) User-friendly tool for power flow analysis and distributed generation optimisation in radial distribution networks. Niger J Technol Dev 14(1):23-33

2. Alsaadi A, Gholami B (2009) An effective approach for distribution system power flow solution. Int J Electr Comp Eng 3(1):1-5

3. Anagnostopoulos PM, Papathanassiou SA (2019) A power flow method for radial distribution feeders with DER penetration. $\mathbf{J}$ Technol Innov Renew Energy 8:1-2

4. Aravindhababu P, Ashokkumar R (2008) A fast decoupled power flow for distribution systems. Electr Power Components Syst 36(9):932-940 
5. Aravindhababu P, Ashokkumar R (2011) A robust decoupled power flow for distribution systems. Energy Convers Manag 52(4):1930-1933

6. Aravindhababu PN, Ganapathy S, Nayar KR (2001) A novel technique for the analysis of radial distribution systems. Int J Electr Power Energy Syst 23(3):167-171

7. Baran ME, Wu FF (1989) Network reconfiguration in distribution systems for loss reduction and load balancing. IEEE Power Eng Rev 9(4):101-102

8. Bazrafshan M, Gatsis N (2017) Comprehensive modeling of threephase distribution systems via the bus admittance matrix. IEEE Trans Power Syst 33(2):2015-2029

9. Bernstein A, Dall'Anese E (2017) Linear power-flow models in multiphase distribution networks. In 2017 IEEE PES Innovative Smart Grid Technologies Conference Europe (ISGT-Europe). 1-6

10. Cespedes RG (1990) New method for the analysis of distribution networks. IEEE Trans Power Deliv 5(1):391-396

11. Chen TH, Yang NC (2010) Loop frame of reference based threephase power flow for unbalanced radial distribution systems. Electr Power Syst Res 80(7):799-806

12. Gianto R (2020) Three-phase distribution system load flow analysis using sequence components. Eur J Electr Eng Comp Sci 4(4):1-5

13. Husain T, Muqueem K, Ansari MM (2016) Power flow analysis of distribution system, international journal of advanced research in electrical electronics and instrumentation. Engineering 5(5):4058-4065

14. Jabr RA (2006) Radial distribution load flow using conic programming. IEEE Trans Power Syst 21(3):1458-1459

15. Kalesar BM, Seifi AR (2010) Fuzzy load flow in balanced and unbalanced radial distribution systems incorporating composite load model. Int J Electr Power Energy Syst 32(1):17-23

16. Kashem MA, Ganapathy V, Jasmon GB (2001) A geometrical approach for network reconfiguration based loss minimization in distribution systems. Int J Electr Power Energy Syst 23(4):295-304

17. Kaushal PK, Tomar MM (2016) Review of load flow analysis for three phase radial distribution system. Int J Eng Techn 2(5):94-99

18. Lin WM, Teng JH (2000) Three-phase distribution network fastdecoupled power flow solutions. Int J Electr Power Energy Syst 22(5):375-380

19. Memon ZA, Trinchero R, Xie Y, Canavero FG, Stievano IS (2020) An iterative scheme for the power-flow analysis of distribution networks based on decoupled circuit equivalents in the phasor domain. Energies. 13(2):386

20. Muruganantham B, Gnanadass R, Padhy NP (2016) Performance analysis and comparison of load flow methods in a practical distribution system. In 2016 National Power Systems Conference (NPSC) 1-6

21. Mythili S, Thiyagarajah K, Rajesh P, Shajin FH (2020) Ideal position and size selection of unified power flow controllers
(UPFCs) to upgrade the dynamic stability of systems: an antlion optimiser and invasive weed optimisation algorithm. HKIE Trans 27(1):25-37

22. Ouali S, Cherkaoui A (2020) An improved backward/forward sweep power flow method based on a new network information Organization for Radial Distribution Systems. J Electr Comp Eng 2020:1-11

23. Pandey A, Jereminov M, Wagner MR, Bromberg DM, Hug G, Pileggi L (2018) Robust power flow and three-phase power flow analyses. IEEE Trans Power Syst 34(1):616-626

24. Rajesh P, Shajin F (2020) A multi-objective hybrid algorithm for planning electrical distribution system. Eur J Electr Eng 22(4-5):224-509

25. Ramana T, Ganesh V, Sivanagaraju S (2013) Simple and fast load flow solution for electrical power distribution systems. Int J Electr Eng Inform 5(3):245

26. Shajin F, Rajesh P (2020) Trusted Secure Geographic Routing Protocol: outsider attack detection in mobile ad hoc networks by adopting trusted secure geographic routing protocol. Int J Pervasive Comp Commun

27. Shakil M, Rashid Z, Hussain GA, Umer F (2020) Power flow analysis and optimization in ring distribution network of Bahawalpur using Newton Raphson method. Indian J Sci Technol 13:2720-2732

28. Shirmohammadi D, Hong HW, Semlyen A, Luo GX (1988) A compensation-based power flow method for weakly meshed distribution and transmission networks. IEEE Trans Power Syst 3(2):753-762

29. Tan Y, Liao C, Li Y, Cao Y, Shahidehpour M, Chen C (2020) A linear power flow model for balanced distribution network with droop-controlled DSTATCOM and voltage controlled DG. Int J Electr Power Energy Syst 117:105665

30. Thota MK, Shajin FH, Rajesh P (2020) Survey on software defect prediction techniques. Int J Appl Sci Eng 17:331-344

31. Venkata Krishna B, Padma Srinivasu N (2019) A direct method for distribution system load flow solutions. Int J Eng Adv Technol 8(6S3):749-753

32. Wu WC, Zhang BM (2008) A three-phase power flow algorithm for distribution system power flow based on loop-analysis method. Int J Electr Power Energy Syst 30(1):8-15

33. Zhang G, Wang C, Wang H, Xie N (2019) On the convergence of the implicit Z-bus power flow method for distribution systems. Electr Power Syst Res 171:74-84

Publisher's Note Springer Nature remains neutral with regard to jurisdictional claims in published maps and institutional affiliations. 